\title{
TAE internal structure through high-resolution soft x-ray measurements in ASDEX-Upgrade
}

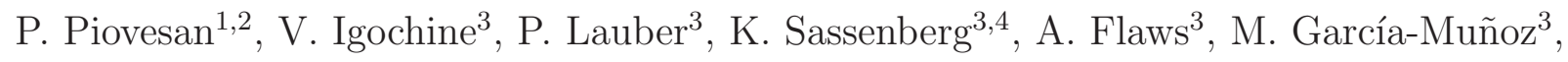 \\ S. Günter ${ }^{3}$, M. Maraschek ${ }^{3}$, L.Marrelli ${ }^{1}$, P. Martinn, ${ }^{1,2}$, P.J. Mc Carthy ${ }^{4}$, and the AUG team \\ 1 Consorzio RFX, Euratom-ENEA Association, \\ Corso Stati Uniti 4, 35127 Padova, Italy \\ 2 Department of Physics, University of Padova, Italy \\ 3 Max-Planck-Institute für Plasmaphysik, \\ EURATOM Association, Garching, Germany \\ 4 Department of Physics, University College Cork, \\ EURATOM-DCU Association, Cork, Ireland and \\ E-mail: paolo.piovesan@igi.cnr.it
}

(Dated: March 17, 2008)

\begin{abstract}
The internal structure of Toroidal Alfvén Eigenmodes (TAEs) excited in ASDEX-Upgrade plasmas with Ion Cyclotron Resonance Heating (ICRH) has been measured through a high-resolution multichord soft x-ray diagnostic. The radial profiles of multiple TAEs are compared with predictions of the linear resistive magnetohydrodynamic code CASTOR and the nonperturbative gyrokinetic code LIGKA and a reasonable good agreement is found. The evolution of the TAE radial profiles could be followed in time, both across sawteeth in pure ICRH discharges and in discharges with different types of transitions from L-mode to H-mode obtained with a combination of ICRH and Neutral Beam Injection.
\end{abstract}

PACS numbers: 52.35.Bj, 52.55.Fa, 52.55.Pi 


\section{INTRODUCTION}

Thermonuclear plasmas are characterized by significant populations of suprathermal particles, i.e. particles with velocity much larger than the thermal velocity [1]. Important examples are fusion-driven $\alpha$ particles and fast ions generated by ion cyclotron resonance heating $(\mathrm{ICRH})$ and neutral beam injection (NBI). Spatial and velocity gradients of the fast particle distribution function may drive magnetohydrodynamic (MHD) instabilities, which to their turn can affect both the fast and thermal particle confinement [2]. Fast particle redistribution or even loss may reduce the current drive and the plasma self-heating efficiency, may have deleterious consequences on the first wall components, and affect the formation of internal transport barriers.

Among fast particle-driven modes, Toroidal Alfvén Eigenmodes (TAEs) play an important role [3]. Due to their relatively high propagation frequency and complex spatial structure, accurate measurements of the TAE radial profiles became possible only recently. Information on the TAE internal structure is crucial to validate the existing theories and make reliable predictions on the impact of TAEs on fast ion confinement in future devices like the International Thermonuclear Experimental Reactor [4, 5].

Initial investigations of the TAE internal structure were made with multichord soft x-ray measurements in DIII-D [6] and ASDEX-Upgrade (AUG) [7]. In both cases the diagnostic resolution was not sufficient to draw definitive conclusions on the comparison with theory. More recently accurate measurements of TAE eigenfunctions were made in DIII-D through fast Electron Cyclotron Emission (ECE), which showed good agreement with predictions of the linear MHD code NOVA [8, 9].

In the last years a strong experimental and theoretical effort has been put in AUG in the investigation of TAEs and other fast particle-driven instabilities [10]. An extensive set of diagnostics is available, including arrays of fast magnetic probes, reflectometry, a newly built fast ion loss detector, and a multichord soft x-ray diagnostic. Here the first results of a study of the TAE internal structure through a multichord soft x-ray diagnostic with high spatiotemporal resolution are presented. This new diagnostic was recently made available after a significant upgrade of a preexisting one $[7,11]$. The improved resolution allowed to measure the radial structure of multiple TAEs simultaneously present and to compare it with predictions of the linear resistive MHD code CASTOR [12] and the nonperturbative gyroki- 
netic code LIGKA [13]. To directly compare the line-integrated soft x-ray measurements and the theoretical predictions, the soft x-ray signals are modeled with the MHD-Interpretation Code [14], using TAE eigenfunctions predicted by CASTOR and LIGKA in input. MHD-IC was developed to fit the effects of rotating and/or growing MHD instabilities on several experimental quantities. A reasonable good agreement among experiment and linear theory predictions was found, as will be shown in the following.

The paper proceeds as follows. In Section II the new multichord soft x-ray diagnostic and the discharges analyzed in this work will be introduced. Section III will present the measurements of the TAE displacement profiles and a comparison with predictions of the linear stability codes CASTOR and LIGKA. The evolution of the TAE profiles across sawteeth and in discharges with different types of transitions from L-mode to $\mathrm{H}$-mode will be described in Section IV and V, respectively. The main conclusions of this work will be summarized in Section VI.

\section{SOFT X-RAY DIAGNOSTIC AND DISCHARGE SETUP}

In the last years the AUG soft x-ray diagnostic was significantly upgraded and presently consists of several fans of lines of sight for a total of 128 channels: the 32 faster channels have $2 \mathrm{MHz}$ sampling frequency and $0.5 \mathrm{MHz}$ bandwidth, while the remaining ones have $0.5 \mathrm{MHz}$ sampling frequency and $0.16 \mathrm{MHz}$ bandwidth. The lines of sight of the three faster cameras are shown in Fig. 1. Only the fast channels are used here, since the TAE frequency in these plasmas falls in the range $180-250 \mathrm{kHz}$, which is higher than the frequency bandwidth of the slower channels.

Two types of plasmas have been considered in the present work: discharges with pure ICRH during the current flat-top (\#21067) and with ICRH in combination with NBI (\#21140). The paper focuses on the two discharges indicated above, whose main waveforms are reported in Figs. 2 and 9, but reproducible results were also found in other similar plasmas. In both cases the ICRH power is ramped up during the first part of the discharge, $1-2 s$, and then maintained constant at about $6 M W$. In discharge \#21140 NBI is also turned on at $t=2.5 \mathrm{~s}$, causing a transition to H-mode, while discharge \#21067 stays in L-mode. ICRH is transiently turned off and replaced by NBI in discharge \#21067 to make possible Motional Stark Effect (MSE) measurements of the core magnetic field, which are 
used to reconstruct the magnetic equilibrium with the CLISTE code [19].

\section{EXPERIMENTAL AND MODELED TAE DISPLACEMENT PROFILES}

Let us start analyzing the pure ICRH discharge \#21067. Fig. 2 shows the Fourier spectrograms of a fast soft x-ray channel viewing the TAE peak with impact parameter $\bar{\rho}_{p o l} \simeq 0.48$ and that of an edge magnetic coil. Here the chord impact parameter $\bar{\rho}_{\text {pol }}$ assumes the value of the poloidal flux coordinate $\rho_{\text {pol }}$ labelling the flux surface, which the chord is tangent to. Multiple TAEs are destabilized above an ICRH power threshold of about 2.5MW. Phase delay analysis of edge magnetic coils at different toroidal angles gives the TAE toroidal mode numbers, which are $n=4,5,6$, as indicated in Fig. 2-(f), with higher mode number corresponding to higher frequency. The TAE frequency is relatively stationary, small variations being associated with density perturbations caused by sawteeth.

Other fast ion-driven modes are visible in the spectrograms: fishbones [16] are present in the $10-40 \mathrm{kHz}$ frequency range, while the peak at about $80 \mathrm{kHz}$ is associated with a core-localized mode recently observed, whose nature is still under investigation [17]. The relation between TAEs and other fast ion-driven modes is also being subject of intense work, but it will not be reported in this paper.

As can be seen in Fig. 2-(d), not all the TAE peaks present in the magnetic spectrograms are also visible in the soft x-ray ones. The cause of this effect is twofold: a particular line of sight may not intersect the TAE eigenfunction, or the TAE frequency peak is hidden by noise, which can be significant at these high frequencies. Cross-correlation with magnetics, which have a much larger signal-to-noise ratio, is used here to filter out the random noise that affects the soft $\mathrm{x}$-ray signals. The soft $\mathrm{x}$-ray fluctuation amplitude of each TAE is thus computed through the following expression [18]:

$$
\tilde{A}_{s x r}=\sqrt{2 \int_{\Delta f} P_{s x r}(f) \gamma_{m a g-s x r}^{2}(f) d f},
$$

where $\gamma_{\text {mag-sxr }}(f)=\left|P_{\text {mag-sxr }}(f)\right| / \sqrt{P_{\text {mag }}(f) P_{\text {sxr }}(f)}$ is the coherence function, $P_{\text {mag }}(f)$ and $P_{s x r}(f)$ the Fourier powerspectra of the magnetic and soft x-ray signals, respectively, $P_{\text {mag-sxr }}(f)$ their cross-powerspectrum, and $\Delta f=5 k H z$ a frequency interval centered on the selected TAE frequency peak. The above quantities are obtained as time averages over 
periods in which all quantities can be considered stationary. The chosen period is divided into $N$ subintervals over which the average is computed, as explained in [18].

The error bars on the soft x-ray fluctuation amplitude defined by Eq. 1 are computed as maximum errors, by propagating the standard deviation of the powerspectrum and coherence, whose expressions are derived in [18]. The maximum and minimum values of the soft x-ray fluctuation amplitude, $\tilde{A}_{s x r}^{ \pm}$, measured by each channel are obtained as follows:

$$
\tilde{A}^{ \pm}=\sqrt{2 \int_{\Delta f} P(f)\left[1 \pm \sigma_{P}(f)\right] \gamma^{2}(f)\left[1 \pm \sigma_{\gamma^{2}}(f)\right] d f},
$$

where the mag and sxr labels are dropped for simplicity. In the above expression $\sigma_{P}(f)=$ $1 / \sqrt{N}$ and $\sigma_{\gamma^{2}}(f)=\sqrt{2}\left[1-\gamma^{2}(f)\right] /[\gamma(f) \sqrt{N}]$ are the relative standard deviation of the powerspectrum and of the coherence squared, respectively. The derivation of these error formulas and more details on the numerical procedure employed to compute the above spectral quantities can be found in [18].

A radial profile of the TAE soft x-ray fluctuation amplitude is obtained by applying the above analysis to the signals measured by the fast lines of sight shown in Fig. 1. In the left column of Fig. 3 are shown the fluctuation amplitude profiles as a function of the chord impact parameter for the $n=4,5,6$ TAEs in discharge \#21067, averaged over the period $t=2.7-3 s$ with $N=32$. This period includes several similar sawtooth crashes. The effect of sawteeth on TAEs is visible in the spectrogram of Fig. 2-(f) as a periodic modulation of the mode amplitude and as small changes in the mode frequency. In the following a more time-resolved analysis will also be reported, which will reveal the evolution of the TAE profile across these sawteeth. In the right column of Fig. 3 are shown the TAE soft x-ray fluctuation amplitude profiles simulated with MHD-IC, with the eigenfunctions predicted by CASTOR in input.

The MHD-IC code receives in input the radial profile of several experimental quantities, such as electron temperature and density, and the magnetic equilibrium reconstructed by CLISTE. The soft x-ray emissivity and its line integrals are computed from these quantities by taking into account the 3D diagnostic geometry, as explained in detail in [14]. The TAE perturbation to the soft x-ray brightness signals is modeled as described in the following. First, the TAE perturbation to the mean electron temperature profile, $T_{e 0}\left(\rho_{p o l}\right)$, is added through the following expression: 


$$
T_{e}\left(\rho_{p o l}, \theta, t\right)=T_{e 0}\left(\rho_{p o l}\right)+\sum_{m=n}^{n+4} \xi_{r}^{m, n}\left(\rho_{p o l}, \theta, t\right) \nabla_{\rho} T_{e 0}\left(\rho_{p o l}\right),
$$

where $\xi_{r}^{m, n}\left(\rho_{\text {pol }}, \theta, t\right)=\hat{\xi}_{r}^{m, n}\left(\rho_{\text {pol }}\right) \cos (m \theta+n \phi-2 \pi f t)$. In this last expression $\hat{\xi}_{r}^{m, n}\left(\rho_{\text {pol }}\right)$ is the $m$ poloidal harmonic of the TAE displacement eigenfunction with toroidal mode number $n ; \theta$ and $\phi$ are the poloidal and toroidal angles, respectively; $f$ is the TAE frequency; and the sum is extended to the five dominant poloidal harmonics. Eq. 3 can be seen as a Taylor series expansion of the electron temperature profile truncated to the $1^{\text {st }}$-order term. This is a very good approximation, since the TAE fluctuation amplitude measured here is very small.

The contribution to the soft x-ray brightness signals from the density perturbation is assumed to be negligible with respect to that from the temperature perturbation, for the following reasons. First, the density profile in these plasmas is much flatter that the temperature profile. Hence the relative density perturbation due to the displacement of this gradient is much lower than the temperature one. This has been checked with dedicated MHD-IC simulations. Second, the dependence of the soft x-ray brightness on the density is weaker than that on the temperature, being proportional to $n_{e}^{2} T_{e}^{\alpha}$, with $\alpha \simeq 3-4$. Third, density fluctuations due to compressional effects, as those identified in [8,9], which may be present even with a flat density profile, are expected to be negligible in these plasmas. In fact compressional effects on the TAE dynamics should be proportional to the coupling of these modes to the slow and fast magnetosonic branches, which is expected to be of order $\beta$. Since $\beta \simeq 1 \%$ is quite low in the discharges analyzed in this work, we may expect this contribution to be small. For these reasons the contribution of the density perturbations to the soft x-ray brightness signal has been neglected. The soft x-ray line integrals are then computed in MHD-IC based on the electron temperature perturbation, Eq. 3, as a function of time after several mode rotation periods.

The radial eigenfunctions $\hat{\xi}_{r}^{m, n}$ used in these MHD-IC simulations are computed with the CASTOR code and are shown in Figs. from 4-(a) to 4 -(c) for the $n=4,5,6$ TAEs. The linear eigenfunction amplitude used in MHD-IC has been rescaled to match the measured soft x-ray fluctuation profiles. The total radial displacement of the $n=4$ TAE obtained from the sum of the poloidal harmonics predicted by CASTOR is also compared with that predicted by LIGKA, as shown in Figs. 4-(d) and 4-(e), respectively, and a good agreement 
is found. Due to its much longer computational time, only the $n=4$ TAE was investigated with LIGKA. The 2D structure of the $n=4$ electron temperature perturbation modelled by MHD-IC with CASTOR eigenfunctions in input is shown in Fig. 5 for discharge \#21067 at $t=2.99 \mathrm{~s}$. A similar plot is also obtained from LIGKA and is not reported here. The 2D plot helps in understanding the line integration effect over the spatial TAE structure.

The radial profile of the soft $\mathrm{x}$-ray brightness fluctuation amplitude is computed from the above simulated signals by integrating the Fourier frequency spectrum around each TAE frequency peak, in a way similar to the experimental case. The results for the $n=4,5,6$ TAEs are shown in the right column of Fig. 3. The simulated profiles are in reasonable good agreement with the experimental ones. This agreement regards the global shape of the TAE eigenfunction, not all its smaller details, as will be better explained in the following. In fact the soft x-ray fluctuation amplitude profile reflects the shape of the TAE eigenfunction, or better of the envelop of the poloidal harmonics forming it. In particular the larger peaks at mid radius, $\bar{\rho}_{\text {pol }} \simeq 0.4-0.7$, are associated with the positive maximum of the TAE eigenfunction, which corresponds to the TAE continuum gap. The negative TAE peak visible in Fig. 4, which is associated with the higher poloidal harmonics, could not be detected, since the soft x-ray brightness becomes very low at $\bar{\rho}_{\text {pol }}>0.7$ in these plasmas, as will be even clearer from the simulations described in the next paragraph.

A test of the soft x-ray diagnostic sensitivity to changes in the shape of the TAE eigenfunction has been made with MHD-IC. The radial profile of the soft x-ray brightness fluctuation amplitude turned out to be very sensitive to the radial position and radial width of the positive TAE peak, thus justifying the use of the soft x-ray diagnostic to investigate the TAE radial profiles. This is shown in Fig. 6, where results of MHD-IC simulations made with analytic eigenfunctions similar in shape to those predicted by CASTOR and LIGKA are reported. Here eigenfunctions with $n=5, m=5,6$ are used, but the same simulations were also carried out for other $n$ values and similar results were found. The shape of the soft x-ray fluctuation profile has been found to depend on the toroidal mode number, but the variation is not significant when $n$ varies from 4 to 6 , as in the experiment. Hence this dependence will not be further discussed here, even though it might be important if a larger range of $n$ values was considered.

By varying the radial width of the eigenfunction in a range $\Delta \rho_{\text {pol }}=0.075-0.3$, the fluctuation profiles change their shape from one peaked off-axis to one peaked in the center, 
as is evident from Fig. 6. For sufficiently small radial widths, $\Delta \rho_{\text {pol }} \lesssim 0.2$, the off-axis peaks in the fluctuation profile correspond to the dominant, positive TAE peak. When the radial width is further increased, the lines of sight viewing the core detect a larger fluctuation amplitude and the fluctuation profile change significantly, eventually becoming peaked in the core. This can be better understood by analyzing the 2D plot of Fig. 5 .

An additional test was made by adding to the previous simulations a negative peak made of higher- $m$ poloidal harmonics, similar to that observed in the CASTOR eigenfunctions of Fig. 4, to test if they may significantly contribute to the fluctuation profile. As noted above, due to the fact that the soft x-ray brightness profile is very low at $\bar{\rho}_{\text {pol }}>0.7$, the contribution of these higher- $m$ harmonics has been verified to be negligible. Based on the above results, it can be concluded that the AUG soft x-ray diagnostic has sufficient resolution to measure the global structure of the TAE eigenfunctions, and in particular the radial position and width of the main TAE peak.

An important feature of the soft x-ray fluctuation amplitude are the sharp jumps in its profile. These are also reproduced in the MHD-IC simulations and are due to a line integration effect. In fact a helical mode with a single poloidal mode number $m$ has a soft x-ray fluctuation profile with $m$ minima and $m+1$ maxima. But, when two or more adjacent poloidal harmonics are summed, as in the TAE case, the profile is a combination of such minima and maxima, and depends on the spatial structure and phase of each individual harmonic. The fine structure of the radial profile can thus be quite complex and it is not possible to fit it with simulated profiles given the present spatial resolution of the diagnostic. Nonetheless, its global features, like the radial position of the TAE peak and its radial width are much more robust, as was shown before, and can be recognized in the experimental data.

The radial displacement profile of each TAE is computed from the soft x-ray fluctuation amplitude profile as $\xi_{r}\left(\bar{\rho}_{p o l}\right)=-\tilde{A}_{s x r} / \nabla_{\bar{\rho}} A_{s x r}$, where $A_{s x r}\left(\bar{\rho}_{p o l}\right)$ is the mean soft x-ray brightness profile. The same method is applied both to the experimental and the simulated data. The comparison between the experimental TAE displacement and the simulated one is reported in Fig. 7 for discharge \#21067. An obvious limit of this method is that the displacement is only defined at chord impact parameters where the radial gradient of the soft x-ray brightness profile is high enough, which occurs usually at $\left|\bar{\rho}_{\text {pol }}\right| \simeq 0.2-0.7$ in the plasmas considered here. The dominant $n=4$ TAE has displacement values in the range $\xi_{r} \simeq 0.1-0.5 \mathrm{~mm}$. The displacement decreases with $n$ and reaches very low values of about 
$0.04 \mathrm{~mm}$ for the $n=6$ mode. The inferred radial magnetic field perturbation in the core varies in a range $\delta b_{r} / B_{0}=\xi_{r} /\left(2 q R_{o}\right) \simeq 0.1-5 \times 10^{-4}$ for the $n=4$ mode, which is similar to the estimates recently made in DIII-D, even though for different types of plasmas [9].

The error bars on the radial displacement are computed by propagating the errors in the soft x-ray fluctuation amplitude and in the radial gradient of the mean soft x-ray brightness, as follows: $\sigma_{\xi}=\sqrt{\sigma_{\tilde{A}}^{2}+\sigma_{\nabla_{\rho} A}^{2}}$. Here $\sigma_{\tilde{A}}^{2}=\max \left(\tilde{A}^{ \pm}\right)$and the error bars in the radial gradient are obtained from a spline fit of the brightness profile.

\section{TAE DYNAMICS ACROSS SAWTEETH}

The sawtooth crash is expected to affect the fast ion profile, which provides the main TAE drive, as well as the safety factor, temperature, and density profiles, which may also modify the gap structure and the TAE damping and driving mechanisms. As shown in Fig. 2-(f), the TAE frequency in discharge \#21067 is slightly modulated by sawteeth. The effect of the sawtooth crash on the TAEs has been studied in this ICRH discharge, by following in time the evolution of their eigenfunction across several sawteeth. To this aim the displacement profile is computed at many subsequent time intervals $1 \mathrm{~ms}$ long, in the same way as described above, by tracking the mode frequency in time. The results of this analysis for the $n=4$ TAE are reported in Fig. 8 .

In the left column of Fig. 8 the magnetic and soft x-ray fluctuation amplitudes associated with the $n=4$ TAE, as well as the radial gradient of the mean soft x-ray brightness and the radial displacement of the $n=4 \mathrm{TAE}$ are plotted as a function of time. The soft x-ray quantities are plotted for three chords with different impact parameter, which are indicated with colored vertical lines in the right column: $\bar{\rho}_{1} \simeq 0.33$ (black line), $\bar{\rho}_{2} \simeq 0.48$ (red line), and $\bar{\rho}_{3} \simeq 0.6$ (blue line). In the right column of Fig. 8 are shown the radial profiles of the same quantities averaged over periods taken just before (green lines) and after (orange lines) seven similar sawtooth crashes. Some of the periods included in this average are indicated with vertical dotted lines in the left column. Figs. 8-(a) and 8-(b) show that the magnetic and soft x-ray fluctuation amplitudes of the $n=4$ TAE have a significant modulation associated with the sawteeth. Both fluctuation amplitudes increase in the period between two sawtooth crashes and sharply decrease at the crash. Nonetheless the radial displacement has a different behavior, as can be seen in Fig. 8-(d) and even better in the radial profiles 
of Fig. 8-(g): after each crash the displacement decreases for impact parameters $\bar{\rho}_{\text {pol }}>0.5$ and increases for $\bar{\rho}_{p o l}<0.5$. In other terms, soon after the crash the TAE eigenfunction is shifted inwards. It is also interesting to observe that the overall TAE displacement does not significantly decrease after the crash, but only its radial structure is modified. This may imply that the redistribution of the fast ion profile is not large, which needs to be verified with further measurements and modeling.

The vertical grey bands in Figs. 8-(c) and 8-(d) indicate time intervals in which the computation of the radial displacement is not reliable, due to the large asymmetry in the mean soft x-ray brightness profiles associated with the sawtooth crash. The error bars in the radial displacement profile in Fig. 8-(g) are computed as explained above. The largest contribution to these error bars is given by the fluctuation profiles, which have a relative error of about $15 \%$, while the error in the radial gradient of the soft x-ray brightness is much lower.

The same analysis was also made for the $n=5,6$ TAEs, but the error bars in these cases were not small enough to let us draw meaningful conclusions. The reason is twofold: the error bars increase when the time interval over which the coherence is computed decreases, as in this case, and the fluctuation amplitude of the $n=5,6$ TAEs is lower than that of the $n=4$ TAE.

\section{TAE DYNAMICS IN DISCHARGES WITH ICRH AND NBI}

Let us now consider discharge \#21140, which is similar to the pure ICRH discharge \#21067 analyzed above, except for the addition of NBI at $t=2.5 s$, as shown in Fig. 9-(b). This discharge exhibits a quite varied TAE dynamics and is well suited to test the soft x-ray diagnostic capabilities in following large variations of the mode frequency and amplitude.

NBI first causes a transition to an ELM-free H-mode lasting from 2.5 to $3 \mathrm{~s}$, as can be seen by the density increase and by the moderate confinement improvement, corresponding to a H-factor relative to the ITER-98 $(y, 2)$ H-mode scaling of about 0.8, as shown in Fig. 9. This discharge is chosen because of the interesting TAE dynamics, but it should be said that it does not reflect the best H-mode confinement performances in AUG. In particular, during the ELM-free H-mode phase, the confinement is limited by a very high total radiation level. NBI has also the effect to increase the TAE frequency and the frequency separation among 
the different TAEs, due to the well-known Doppler shift effect described in [15].

Due to switch-off problems of the ICRH system, the ELM-free H-mode is transiently lost in the period from 3 to $3.7 \mathrm{~s}$. An ELMy H-mode with a slightly lower H-factor of 0.7 is then recovered, when the ICRH system starts working properly again. A $(m=2, n=1)$ mode grows in this second H-mode phase and eventually locks to the wall, as is visible from the locked-mode indicator of Fig. 9-(e). This last quantity is defined as the difference between two saddle coils $180^{\circ}$ toroidally apart measuring the radial field of an odd component mode. Values of the locked-mode indicator exceeding \pm 0.15 indicate that a locked mode is present and information from magnetic coils indicate a $(2,1)$ helicity. The locked mode very likely contributes to limit the confinement performance in this phase. Moreover the braking exerted by the mode on the plasma may explain the reduction of the TAE frequencies relative to the previous H-mode period, as is visible in Fig. 9-(f), which is due to the above mentioned Doppler shift effect.

The time-averaged radial displacement profiles of the $n=4,5,6$ TAEs are computed in the three periods illustrated above, with different confinement properties and TAE behavior, and the results are shown in Fig. 10: the L-mode profiles are shown in the left column, the ELM-free H-mode profiles in the central column and the ELMy H-mode profiles in the right column. The time intervals over which the average is computed are indicated in the Figure. The maximum displacement tends to decrease with the toroidal mode number for the Lmode and ELM-free H-mode cases, as observed above also for the pure ICRH discharge \#21067. The situation is different during the ELMy H-mode period, when the $n=5$ TAE tends to dominate over the other modes.

The average displacement value of all the TAE modes increases by about a factor of three when going from L-mode to ELM-free H-mode conditions and by about a factor of four from ELM-free to ELMy H-mode. The ELMy H-mode displacement is also one order of magnitude higher than the values reported in Fig. 7 for discharge \#21067, which is in L-mode. The TAE amplitude increase during the first transition may be explained by a stronger drive of the mode due to larger electron temperature and/or to a better fast ion confinement associated with the H-mode conditions. The further increase of the mode amplitude during the ELMy H-mode phase instead cannot be explained by these effects, since both the electron temperature and the H-factor are not varying significantly with respect to the previous H-mode period. Nonetheless, it can be noted that the density is 
lower in this phase, which may cause a longer fast ion slowing down time. Since the ICRH and NBI power is constant, this would imply a larger fast ion population and hence a larger TAE drive.

The effect of the edge density profile on the gap structure and the TAE stability in the two H-mode phases described above has been also investigated. AUG has a unique diagnostic set to measure the edge density profile, combining very precise reflectometry and Lithium beam measurements [10]. Data from both diagnostics have been fitted to provide a wellconstrained input to CLISTE and CASTOR. These simulations have shown that the edge gap is closed in both phases and the mode growth rate does not change significantly. An effect of the edge density profile in the large TAE amplitude increase described above can be thus ruled out. As commented above, such an effect may be ascribed to an increase in the fast ion drive. A further possibility is a modification of the core damping, which cannot be evaluated very precisely at the moment due to uncertainties in the value of the cental safety factor.

Further simulations of the TAE damping and driving mechanisms in all the phases studied above, as well as simulations of the fast ion profiles produced by ICRH and NBI, are needed and may explain more quantitatively the large variations of the TAE eigenfunctions observed in this discharge. It should be also noted that the effect of the $(2,1)$ locked mode on the fast ion population may be significant in this phase, as recent measurements made in similar AUG plasmas suggest [20]. This effect should be taken into account in a more detailed investigation of this discharge, which is beyond the scope of this paper.

\section{CONCLUSIONS}

In conclusion, a significant upgrade of the AUG multichord soft x-ray diagnostic allowed to measure the radial structure of multiple TAEs in ICRH plasmas and to compare it with the predictions of two linear codes, the resistive MHD code CASTOR and the nonperturbative gyrokinetic code LIGKA. A reasonable good agreement among experiment and theoretical predictions was found, which confirms the validity of linear theory in conditions near marginal stability. The TAE profiles can be followed in time across sawteeth and during different types of transitions from L-mode to H-mode produced by combining ICRH and NBI, and an interesting new phenomenology could be observed. Ongoing work is dedicated 
to a more thoroughly comparison with theory and to a further upgrade of the soft x-ray diagnostic.

Acknowledgement. This work was supported by the European Communities under the contract of Association between EURATOM/ENEA. The views and opinions expressed herein do not necessarily reflect those of the European Commission.

[1] Heidbrink W.W. and Sadler G.J. 1994 Nucl. Fusion 34535

[2] Chen L. and Zonca F. 2007 Nucl. Fusion 47 S727

[3] Wong K.L. 1999 Plasma Phys. Control. Fusion 41 R1

[4] ITER Physics Expert Group on Energetic Particles, Heating, and Current Drive et al, 1999 Nucl. Fusion 392471

[5] Fasoli A. et al 2007 Progress in the ITER Physics Basis Nucl. Fusion 47 S264

[6] Carolipio E.M., Heidbrink W.W., Cheng C.Z., Chu M.S., Fu G.Y., Jaun A., Spong D.A., Turnbull A.D. and White R.B. 2001 Phys. Plasmas 83391

[7] Borba D., Conway G.D., Günter S., Huysmans G.T.A., Klose S., Maraschek M., Mück A., Nunes I., Pinches S.D., Serra F. and the ASDEX Upgrade Team 2004 Plasma Phys. Control. Fusion 46809

[8] Van Zeeland M.A., Kramer G.J., Austin M.E., Boivin R.L., Heidbrink W.W., Makowski M.A., McKee G.R., Nazikian R., Solomon W.M. and G. Wang 2006 Phys. Rev. Lett. 97135001

[9] Van Zeeland M.A., Austin M.E., Gorelenkov N.N., Heidbrink W.W., Kramer G.J., Makowski M.A., McKee G.R., Nazikian R., Ruskov E. and Turnbull A.D. 2007 Phys. Plasmas 14056102

[10] Günter S. et al 2007 Nucl. Fusion 47920

[11] Mück A. 2004 Study of the sawtooth instability and its control in the ASDEX Upgrade tokamak Ph.D. Thesis Technische Universität, Munich, Germany

[12] Huysmans G.T.A., Goedbloed J.P. and Kerner W. 1993 Phys. Fluids B5 1545

[13] Lauber Ph., Günter S. and Pinches S.D. 2005 Phys. Plasmas 12122501

[14] Igochine V., Günter S. and Maraschek M. 2003 Nucl. Fusion 431801

[15] Strait E.J., Heidbrink W.W. and Turnbull A.D. 1994 Plasma Phys. Control. Fusion 361211

[16] Kass T., Bosch H.-S., Hoenen F., Lackner K., Maraschek M., Zohm H. and the ASDEX Upgrade Team 1998 Nucl. Fusion 38807 
[17] García-Muñoz M., Fahrbach H.-U., Günter S., Igochine V., Mantsinen M.J., Maraschek M., Martin P., Piovesan P., Sassenberg K. and Zohm H. 2008 Phys. Rev. Lett. 100055005

[18] Bendat J.S. and Piersol A.G. 2000 Random Data (Wiley: New York)

[19] Mc Carthy P.J. 1999 Phys. Plasmas 63554

[20] García-Muñoz M., Martin P., Fahrbach H.-U., Gobbin M., Günter S., Maraschek M., Marrelli L., Zohm H. and the ASDEX Upgrade Team 2007 Nucl. Fusion 47 L10 


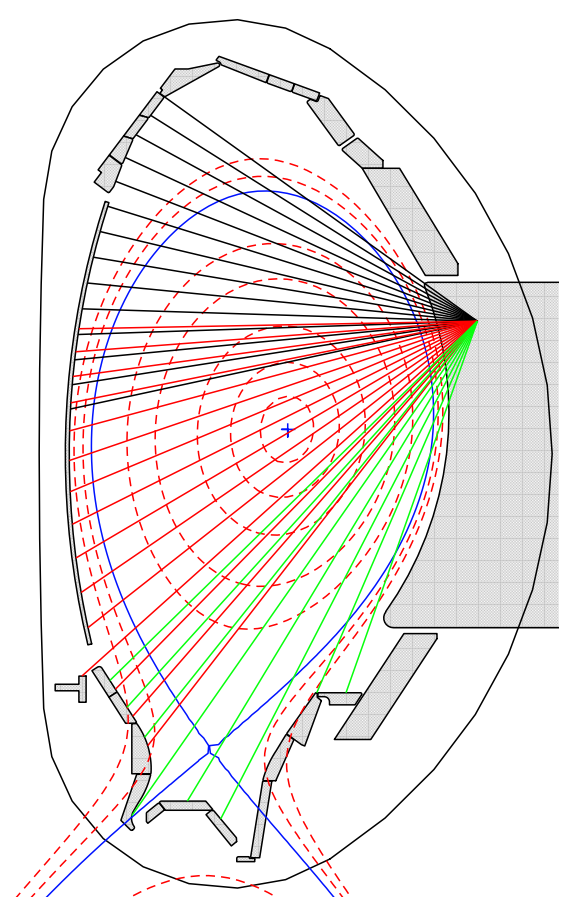

FIG. 1: AUG poloidal cross-section with lines of sight of the fast soft x-ray camera. Dashed lines represent contour levels of the safety factor for discharge \#21067 at $t=2.99 \mathrm{~s}$. 

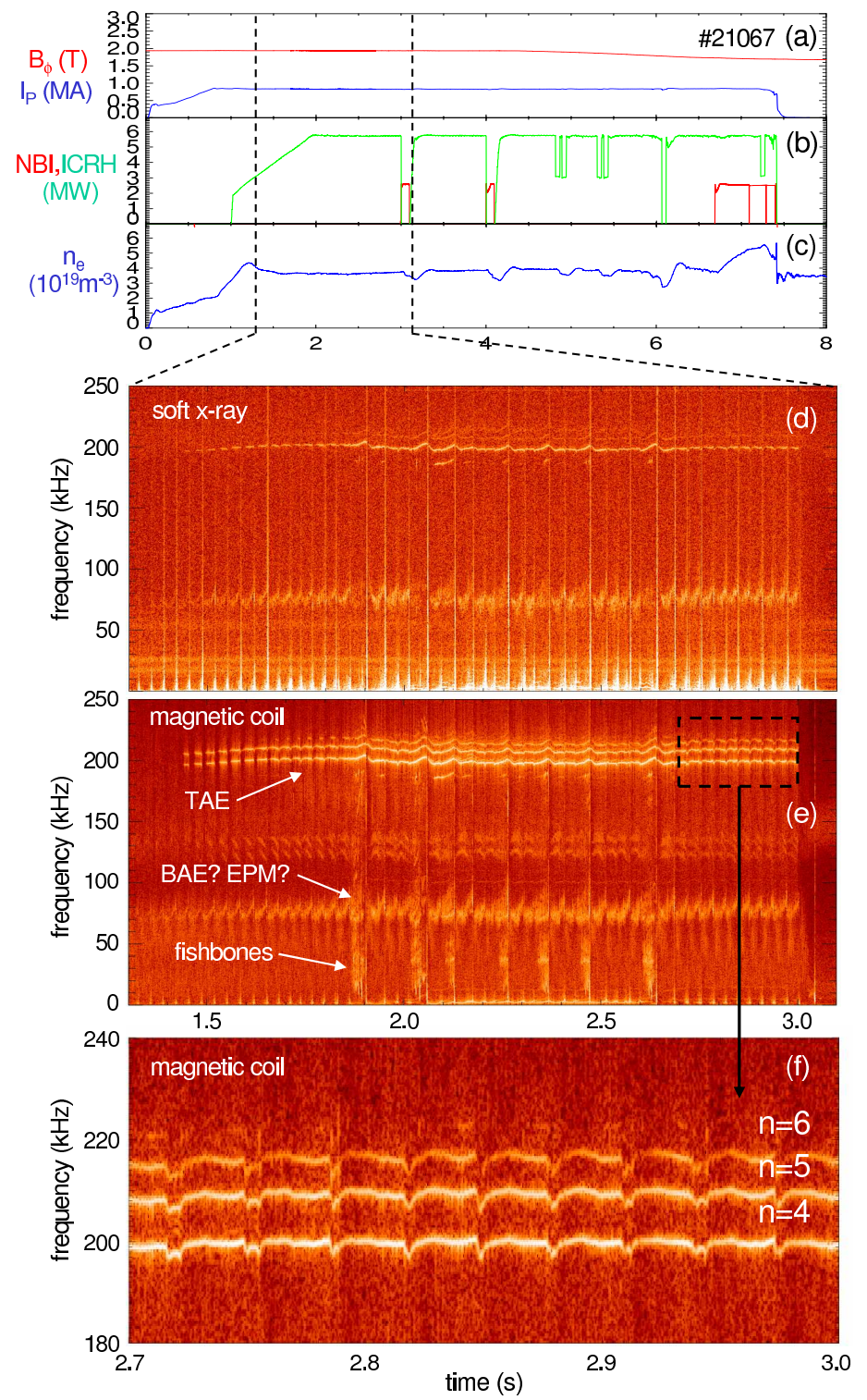

FIG. 2: Main waveforms of the pure ICRH discharge \#21067: (a) plasma current and toroidal magnetic field, (b) ICRH and NBI power input, (c) line-integrated core electron density. Fourier spectrograms of (d) a soft x-ray channel intersecting the TAE peak and (e)-(f) of a magnetic coil. 


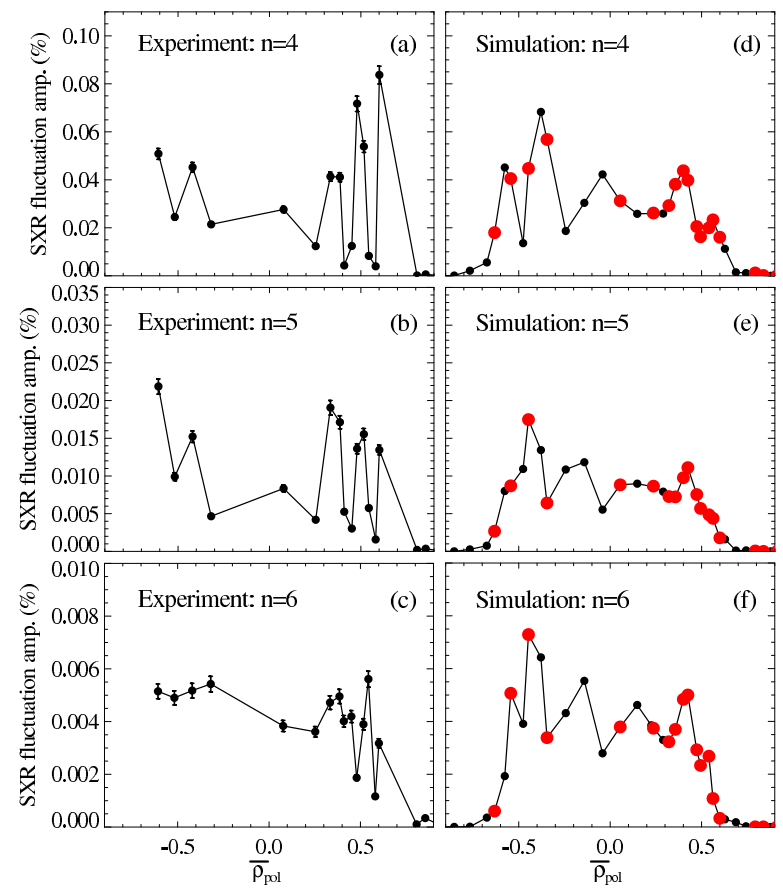

FIG. 3: Radial profiles of the soft x-ray brightness fluctuation amplitude of the $n=4,5,6$ TAEs in discharge \#21067, averaged over the period $t=2.7-3 s$. (a)-(c) Experimental and (d)-(f) simulated profiles are compared. Red dots indicate the channels actually available in discharge \#21067. 

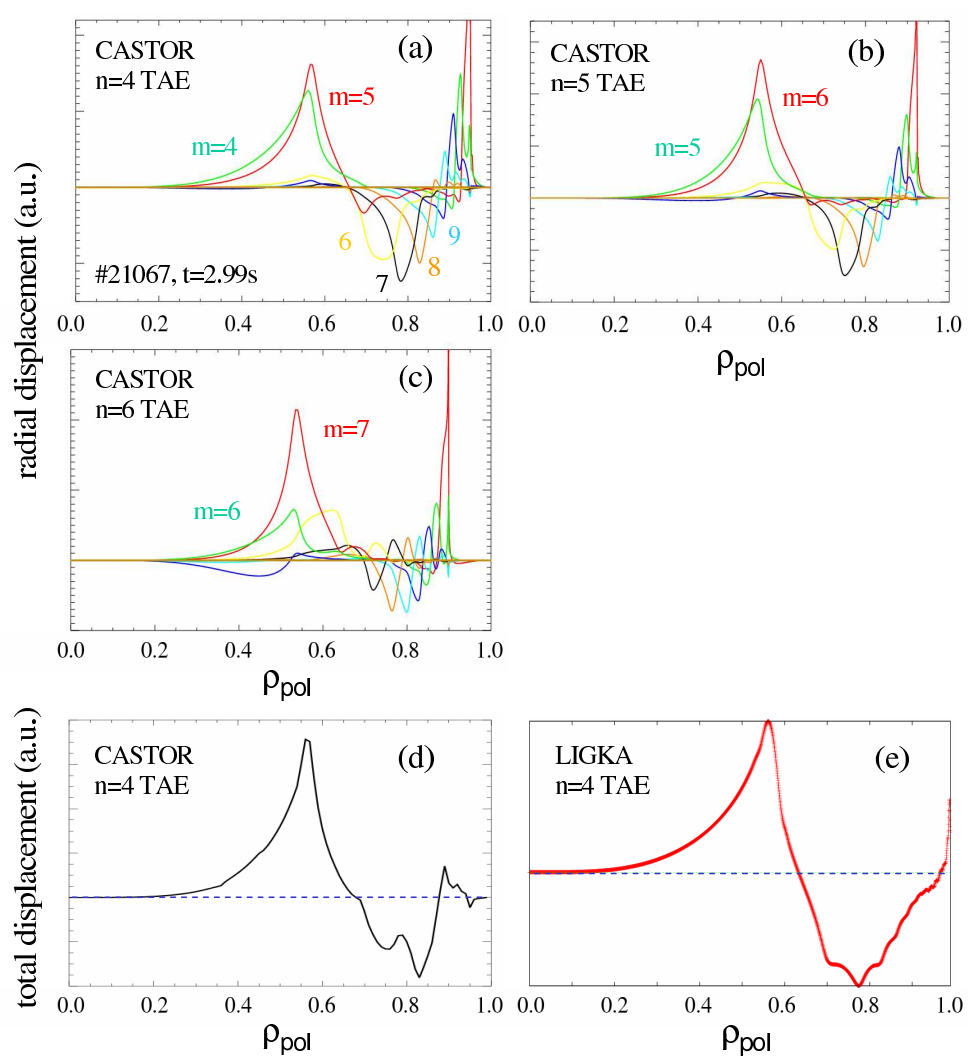

FIG. 4: (a)-(c) Radial eigenfunctions of the $n=4,5,6$ TAEs predicted by CASTOR in discharge \#21067 at $t=2.99 \mathrm{~s}$ and total radial displacement computed by (d) CASTOR and (e) LIGKA for the $n=4$ TAE in the same case. 


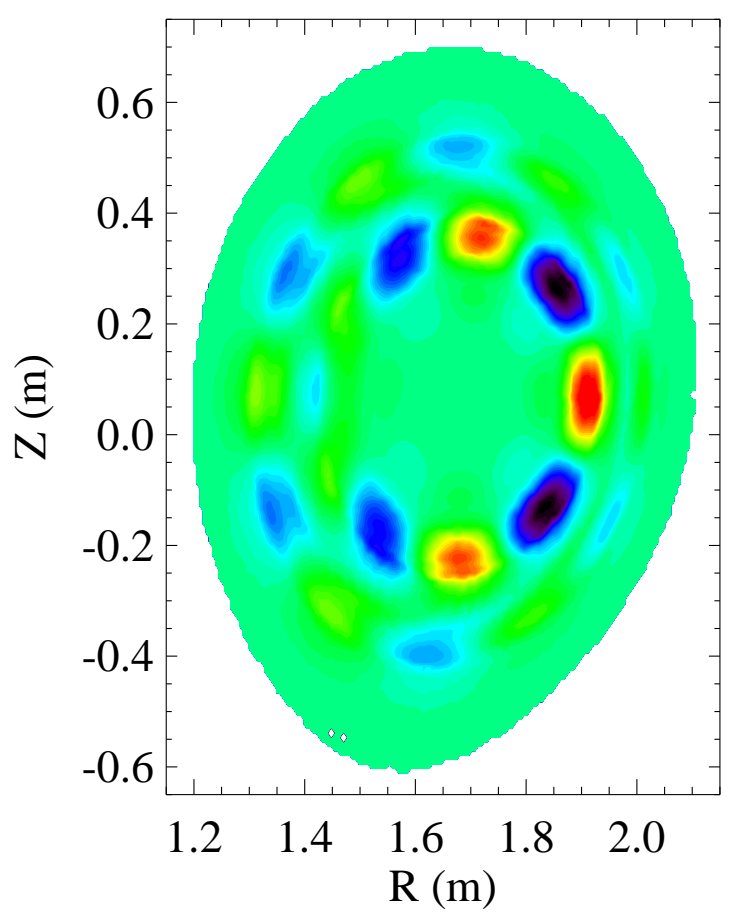

FIG. 5: Poloidal cross-section of the electron temperature perturbation due to the $n=4$ TAE in discharge 21067 at $t=2.99 \mathrm{~s}$, as predicted by MHD-IC simulations with CASTOR eigenfunctions in input. 

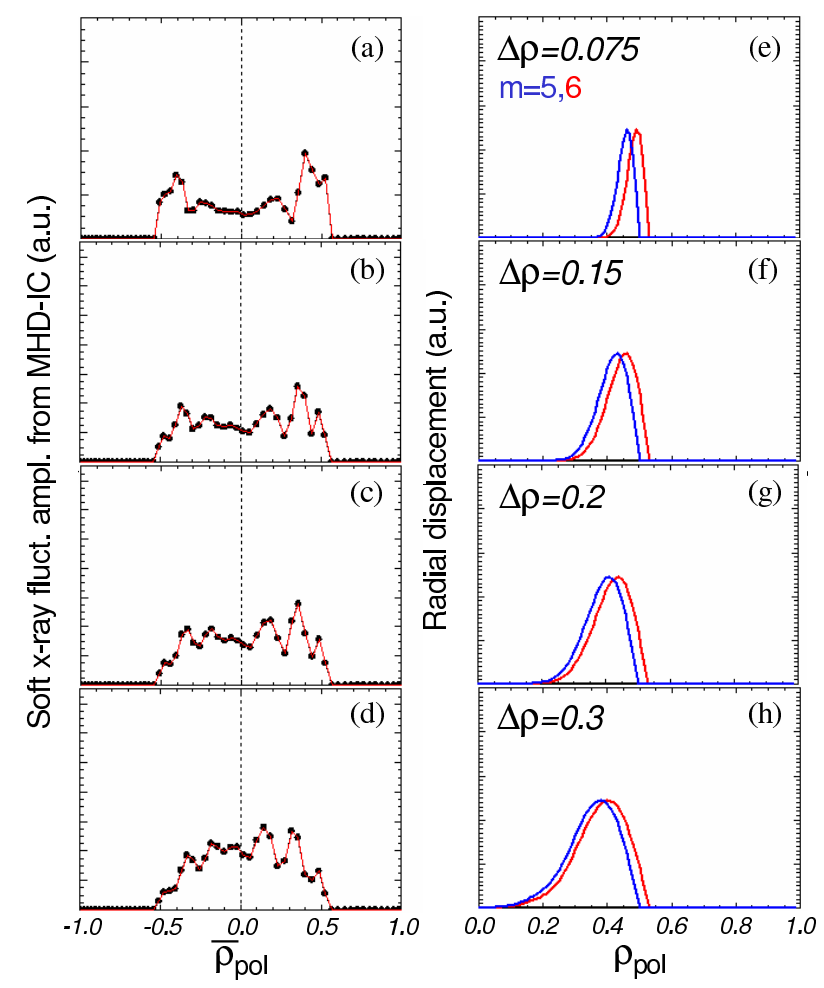

FIG. 6: (a)-(d) Radial profiles of the soft x-ray brightness fluctuation amplitude simulated with MHD-IC using in input (e)-(h) analytical eigenfunctions with $n=5, m=5,6$ and increasing radial width. 


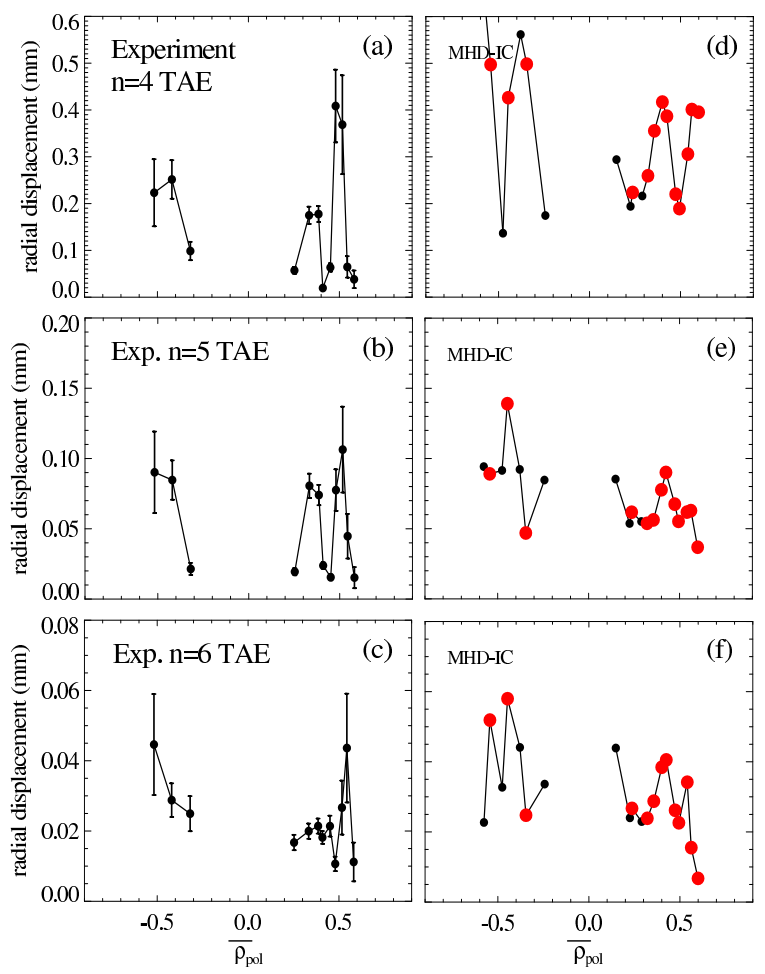

FIG. 7: Radial profiles of the $n=4,5,6$ TAE displacement in discharge $\# 21067, t=2.7-3 s$. Experimental profiles (left column) are compared with profiles simulated with MHD-IC (right column) using in input the CASTOR eigenfunctions reported in Fig. 4. Red dots indicate the channels actually available in discharge \#21067. 

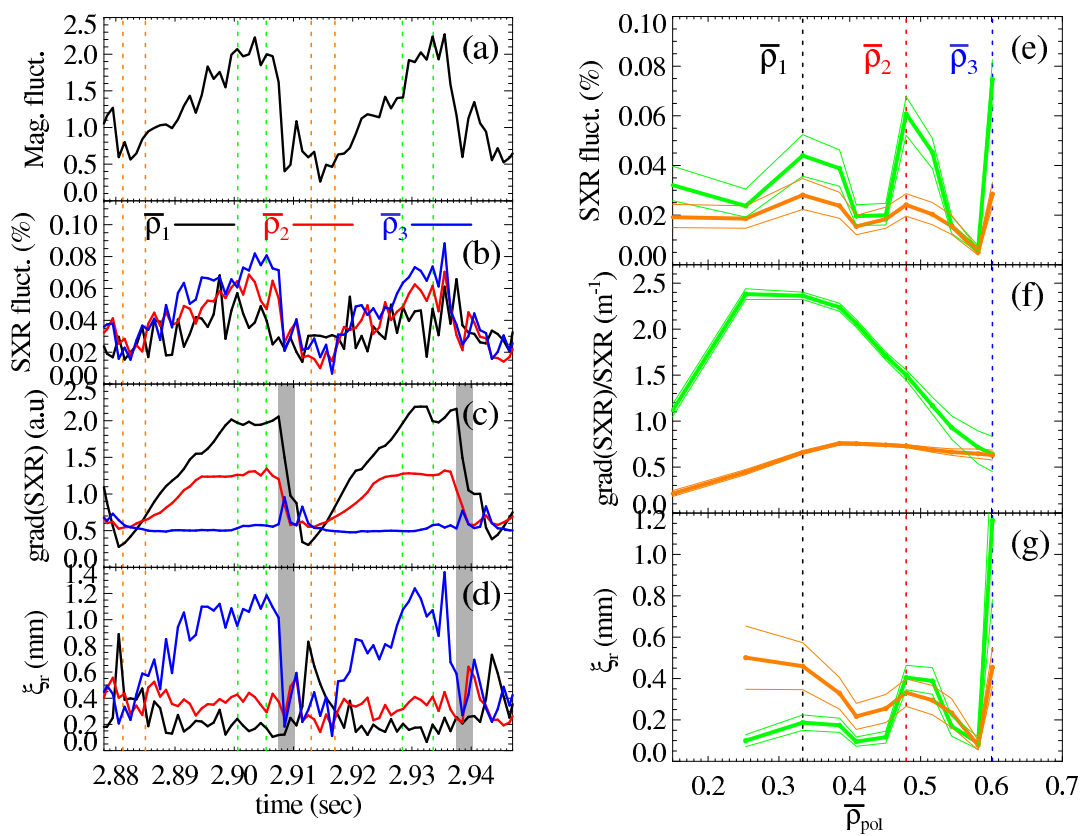

FIG. 8: (a)-(d) Magnetic and soft x-ray fluctuation amplitude, radial gradient of the soft x-ray brightness and radial displacement of the $n=4$ TAE as a function of time across sawteeth in discharge \#21067. Vertical grey bands indicate periods during the crash, when the computation of the displacement is not reliable. (e)-(g) Radial profiles of the same quantities averaged over periods taken just before (green lines) and after (orange lines) seven similar sawtooth crashes. Some of the periods included in the average are indicated with vertical dotted lines in the left column. 


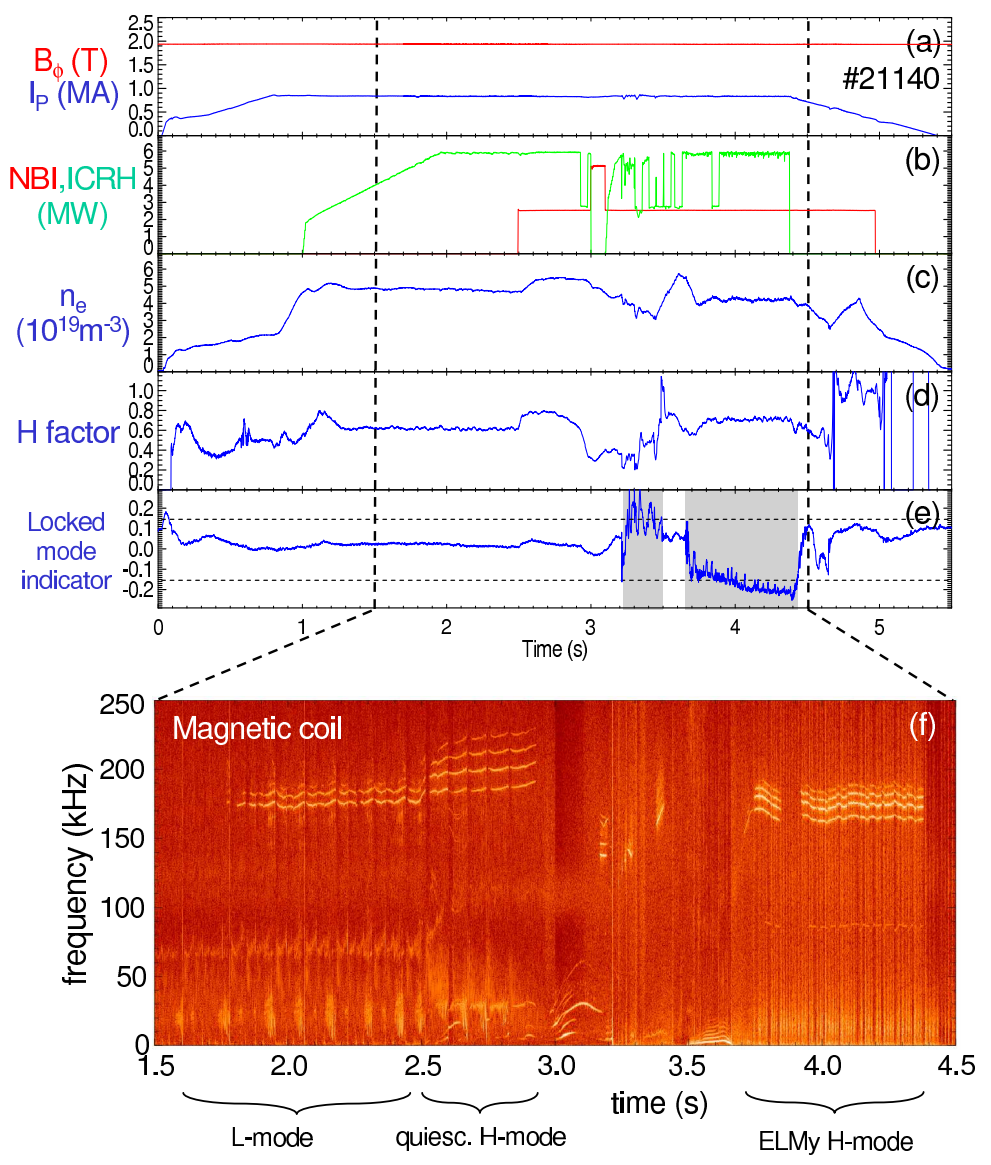

FIG. 9: Main waveforms of discharge \#21140: (a) plasma current and toroidal magnetic field, (b) ICRH and NBI power input, (c) line-integrated core electron density, (d) H-factor relative to the ITER-98 $(y, 2)$ H-mode scaling, and (e) locked mode indicator, where the grey bands indicate periods when a $(2,1)$ locked mode is present. (f) Fourier spectrogram of a magnetic coil. 

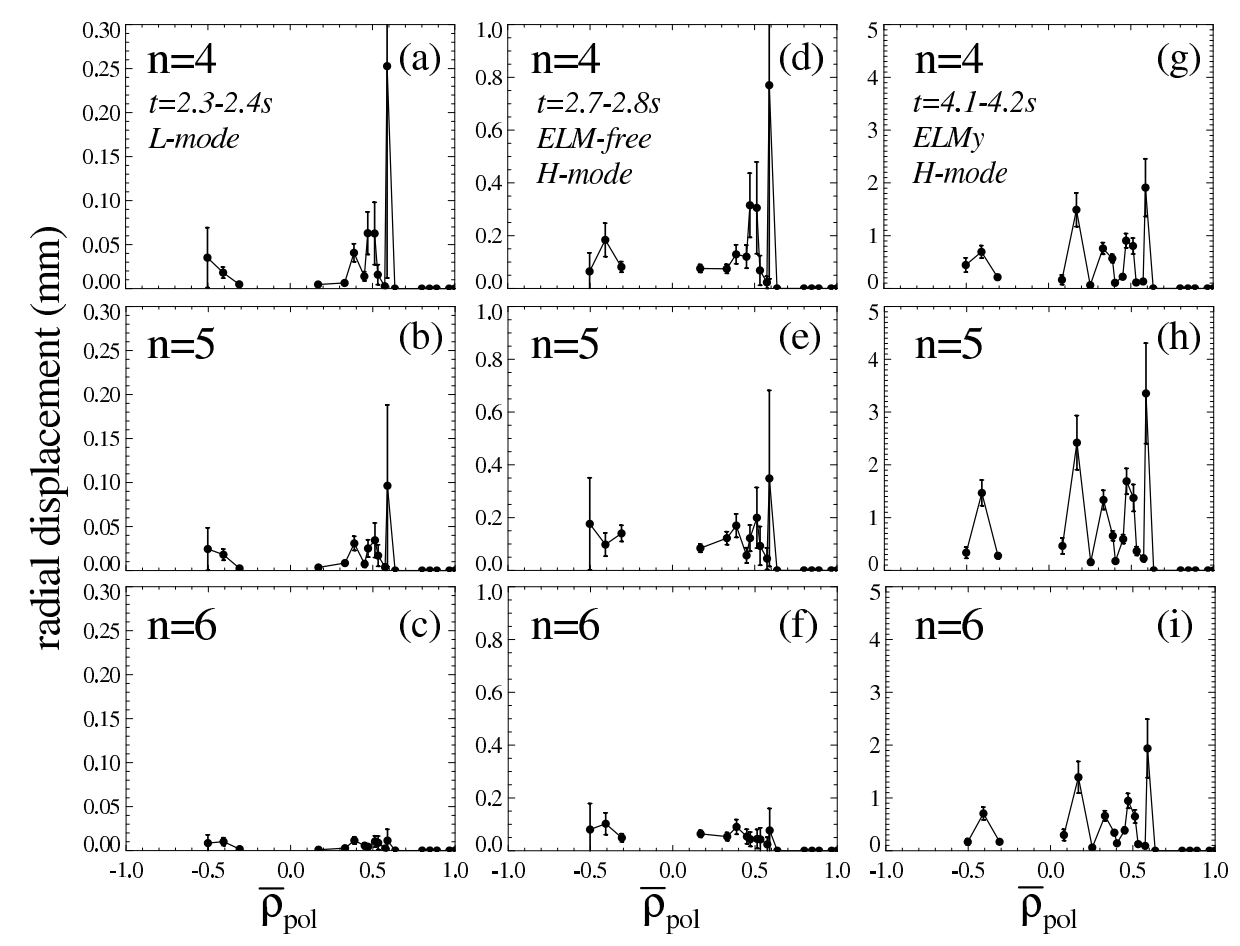

FIG. 10: Time-averaged radial profiles of the $n=4,5,6$ TAE displacement in three periods of discharge \#21140: (a)-(c) during L-mode, (d)-(f) ELM-free H-mode, and (g)-(i) ELMy H-mode. The periods over which the average is computed are indicated in the plots. 